\title{
Comparison between X-ray morphology and optical characteristics of clusters of galaxies
}

\author{
Yasuhiro Hashimoto $^{1}$, J. P. Henry ${ }^{1,2}$, H. Boehringer ${ }^{1}$, and G. Hasinger ${ }^{1}$ \\ 1 Max-Planck-Institut für extraterrestrische Physik, Giessenbachstrasse 85748 Garching, Germany \\ e-mail: hashimot@mpe.mpg.de \\ 2 Institute for Astronomy, University of Hawaii, 2680 Woodlawn Drive, Honolulu, Hawaii 96822, USA
}

Received 28 November 2006 / Accepted 22 February 2007

\section{ABSTRACT}

\begin{abstract}
Aims. We quantitatively studied the X-ray cluster morphology, and then investigated the relationships between the cluster X-ray morphology and various cluster characteristics determined from the optical data.

Methods. Using a sample of 101 clusters at redshift $z \sim 0.05-1$ taken from the Chandra archive, the X-ray morphology is characterized by a series of objectively-measured simple statistics of X-ray surface brightness, which are designed to be robust against variations of image quality caused by various exposure times and various cluster redshifts.

Results. The main conclusions from this work are: (1) X-ray morphologies of clusters show no significant correlation with optical richness, or related quantities. (2) X-ray morphologies of clusters are significantly correlated with the degree to which the brightest cluster member stands out against the cluster background. (3) X-ray morphologies of clusters are also correlated with optical concentration defined by galaxy distribution including fainter populations. (4) The ratio of the strong-lensing to X-ray mass measurements is correlated with the X-ray concentration, in that highly X-ray concentrated clusters show compatible strong-lensing and X-ray mass measurements, while those with low X-ray concentration show discrepancy between the two mass measurements.
\end{abstract}

Key words. galaxies: clusters: general - X-rays: galaxies: clusters - galaxies: evolution

\section{Introduction}

The important connection between the morphologies of galaxy clusters and cosmological parameters has received much recent attention (Richstone et al. 1992; Evrard et al. 1993; Mohr et al. 1995). This connection has generally been formulated in terms of the frequency of "substructure" in clusters. From qualitative measures of the frequency of substructure in clusters, investigators have attempted to determine $\Omega_{\mathrm{m}}$ (e.g. Richstone et al. 1992) and the power spectrum of primordial density fluctuations (e.g. David et al. 1993) by comparison to Press \& Schechter (1974) type predictions of the distribution of collapsed objects.

Methods to quantify structures at optical wavelengths have mostly used both the distribution of cluster galaxies, and lensing. The distribution studies analyze, either visually or objectively, the substructure in $1 \mathrm{D}, 2 \mathrm{D}$ or sometimes $3 \mathrm{D}$, depending on the level of information available (e.g. Geller \& Beers 1982; Dressler \& Shectman 1988; Rhee et al. 1991; Bird 1994; Kriessler \& Beers 1997). Bautz \& Morgon (1970) classified cluster morphology based on visual inspection of optical images, by the degree to which the brightest cluster member stands out against the cluster background. The RS system (Rood \& Sastry 1971; Struble \& Rood 1982, 1984, 1987) is based on the projected distribution of the brightest galaxies in the cluster. The RS system is composed of six major classes: cD, B, C, L, F, and I-type clusters. These six classes have been interpreted as corresponding to a sequence of cluster evolution (Forman \& Jones 1982; Struble \& Rood 1982, 1984). Butcher \& Oemler (1985) characterized the optical morphology of cluster by the degree of central concentration of galaxy distribution. Their concentration is defined by $\log \left(R_{60} / R_{20}\right)$ where $R_{60}$ or $R_{20}$ is the radius of the circle containing $60 \%$ or $20 \%$ of the clusters's projected galaxy distribution, respectively.

An alternative method comes from X-ray wavelengths, because cluster mergers compress and heat the intracluster gas, and this can be measured as distortions of the spatial distribution of X-ray surface brightness and temperature. Jones \& Forman (1999) visually examined 208 clusters observed with Einstein X-ray satellite and separated these clusters into six morphological classes. Meanwhile, using the Einstein images, Mohr et al. (1995) measured emission-weighted centroid variation, axial ratio, orientation, and radial falloff for a sample of 65 clusters, while several other studies used ellipticity (e.g. McMillan et al. 1989; Gomez et al. 1997; Kolokotronis et al. 2001; Melott et al. 2001; Plionis 2002). Buote \& Tsai $(1995,1996)$ used a power ratio method for 59 low redshift clusters observed with ROSAT, and Jeltema et al. (2005) have extended the method to 40 clusters at $z=0.15-0.9$ using Chandra data. Schuecker et al. (2001) conducted a study of 470 clusters from the ROSAT-ESO FluxLimited X-ray (REFLEX) cluster survey (Böhringer et al. 2000), using sophisticated statistics, such as Fourier elongation test, Lee test, and $\beta$ test. Hashimoto et al. (2006) studied X-ray cluster morphology using a sample of 101 clusters of galaxies at redshift $z \sim 0.05-1$ taken from the Chandra archive. There, X-ray morphology is quantitatively characterized by a series of objectively measured simple statistics, such as concentration, asymmetry, elongation, and off-centerness of the X-ray surface brightness distribution. These measures are designed to be robust against variations of image quality caused by various exposure times and various cluster redshifts.

Unfortunately, quantifying cluster structure by investigating the distribution of cluster galaxies in optical wavelengths 
requires a large number of galaxies, and is more susceptible to contamination from foreground and background objects. Lensing study in optical wavelengths is also sensitive to this contamination, and does not have good spatial resolution except for the central region of a cluster. The X-ray method is superior against for/background because X-ray emissivity is proportional to the square of the electron density, and therefore less affected by the superposed structures than optical data. Meanwhile, the advantage of using optical data is the size of the available cluster catalogs, which can be much larger than those originating from $\mathrm{X}$-ray data. By the systematic comparison between X-ray and optical methods, one may calculate and calibrate different biases using the X-ray data with which we could measure and test easily similar substructure diagnostics in a large, optical data set.

Despite this close and complementary nature between the $\mathrm{X}$-ray and optical characteristics of clusters, not much has been known about the quantitative relationship between the X-ray and optical morphologies of a cluster. There are several studies comparing the X-ray and the optical characteristics of clusters (e.g. Donahue et al. 2001; Yee \& Ellingson 2003; Popesso et al. 2004). However, these studies are all comparing X-ray and optical characteristics related essentially to the cluster richness or mass, and not related to cluster morphology or its distortion. Thus, despite their importance, these studies are potentially insensitive to, and their mass comparisons are possibly contaminated by, the various degrees of cluster dynamical state.

Because of this need to measure the cluster dynamical state in both optical and X-ray wavelengths, Mohr et al. (1993) and Rizza et al. (1998) have investigated cluster substructure using optical and X-ray data, in a complementary fashion. Kolokotronis et al. (2001), using 22 very rich low redshift clusters of galaxies, have compared cluster morphologies using ROSAT images and optical images, by measuring centroid shift, ellipticity, and ellipsoidal position angle. They discovered that many clusters show significant alignment between the optical and X-ray position angle, but other measures show only weak to null correlations between the optical and X-ray wavelengths. Moreover, $\sim 22 \%$ of their clusters seem to have distinct substructures in the optical which are not verified in the X-ray images, and thus are suspected of being due to optical projection effects. Despite their important implications, previous studies are unfortunately limited to rather a small number of substructure measures. Samples are also limited only to very rich clusters at low redshift, partially because of the limited capability of ROSAT satellite, and this hinders systematic and comprehensive comparisons between the X-ray and optical morphologies over a wide range of cluster properties.

Here we report our systematic and quantitative comparison between the cluster X-ray morphology and the optical morphology, and other characteristics. Using a sample of 101 clusters at redshift $z \sim 0.05-1$ taken from the Chandra archive, the $\mathrm{X}$-ray morphology is quantitatively characterized by a series of objectively-measured simple statistics of X-ray surface brightness, which are designed to be robust against variations of image quality caused by various exposure times and various cluster redshifts. X-ray morphology and optical characteristics are then quantitatively compared.

\section{The X-ray sample and $X$-ray measures}

Here we briefly summarize our X-ray sample, data preparation, and X-ray measures. More detailed descriptions can be found in Hashimoto et al. (2006).
Almost all clusters are selected from flux-limited X-ray surveys, and X-ray data are taken from the Chandra ACIS archive. A lower limit of $z=0.05$ or 0.1 is placed on the redshift to ensure that a cluster is observed with sufficient field-of-view with ACIS-I or ACIS-S, respectively. The majority of our sample comes from the ROSAT Brightest Cluster Sample (BCS; Ebeling et al. 1998), and the Extended ROSAT Brightest Cluster Sample (EBCS; Ebeling et al. 2000). When combined with EBCS, the BCS clusters represent one of the largest and most complete $\mathrm{X}$-ray selected cluster samples, which is currently the most frequently observed by Chandra. As of 2005 October, 55 BCS + 13 EBCS (hereafter BCS) clusters with $z>0.05$ (ACIS-I), or $z>0.1$ (ACIS-S), are publicly available in the Chandra archive. Additionally we included all clusters from the X-ray flux limited sample of Edge et al. (1990) at $z>0.05$ or 0.1 not in the BCS that were observed with the Chandra ACIS.

To extend our sample to higher redshifts, additional high- $z$ clusters are selected from various deep surveys; 10 of these clusters are selected from the ROSAT Deep Cluster Survey (RDCS: Rosati et al. 1998), 10 from the Einstein Extended Medium Sensitivity Survey (EMSS; Gioia et al. 1990; Henry et al. 1992), 14 from the 160 Square Degrees ROSAT Survey (Vikhlinin et al. 1998), 2 from the Wide Angle ROSAT Pointed Survey (WARPS; Perlman et al. 2002), and 1 from the North Ecliptic Pole survey (NEP; Henry et al. 2001), RX J1054 was discovered by Hasinger et al. (1998), RX J1347 was discovered in the ROSAT All Sky Survey (Schindler et al. 1995), and 3C295 has been mapped with Einstein (Henry \& Henriksen 1986).

The resulting sample we processed contains 120 clusters. At the final stage of our data processing, to employ our full analysis, we further applied a selection based on the total counts of cluster emission, eliminating clusters with very low signalto-noise ratio. Clusters whose center is too close to the edge of the CCD are also removed. The resulting final sample contains 101 clusters with redshifts between $0.05-1.26$ (median $z=0.226$ ), and luminosity between $1.0 \times 10^{44}-1.2 \times 10^{46} \mathrm{erg} / \mathrm{s}$ (median $8.56 \times 10^{44} \mathrm{erg} / \mathrm{s}$ ).

We removed the point sources, except for a source at the center of the cluster which was mostly the peak of the surface brightness distribution rather than a real point source. The images were then smoothed with Gaussian $\sigma=5^{\prime \prime}$. We decided to use isophotal contours to characterize an object region, instead of a conventional circular aperture, because we did not want to introduce any bias in the shape of an object. To define constant metric scale to all clusters, we adjusted an extracting threshold in such a way that the square root of detected object area times a constant was $0.5 \mathrm{Mpc}$, i.e. const $\sqrt{\text { area }}=0.5 \mathrm{Mpc}$. We chose to use the const $=1.5$, because the isophotal limit of a detected object was best represented by this value.

The morphology of cluster X-ray emission is then characterized objectively, by various morphological measures described in Hashimoto et al. (2006) (see also Hashimoto et al. 1998), such as, centroid, centered-second moments, ellipticity, off-center measure, concentration, and asymmetry. Centroid and centered-second moments are computed using the first and second order moments of the profile, while the ellipticity is simply defined by the ratio of semi-major and semi-minor axis lengths. The degree of off-center is determined by the distance between the centroid and maximum intensity peak, while the degree of concentration of the surface brightness profile is is defined by the ratio between central $30 \%$ and whole $100 \%$ elliptical apertures. Finally, asymmetry is measured by first rotating a galaxy image by 180 degrees around the image center, then subtracting the rotated image from the original unrotated one. The residual signals 

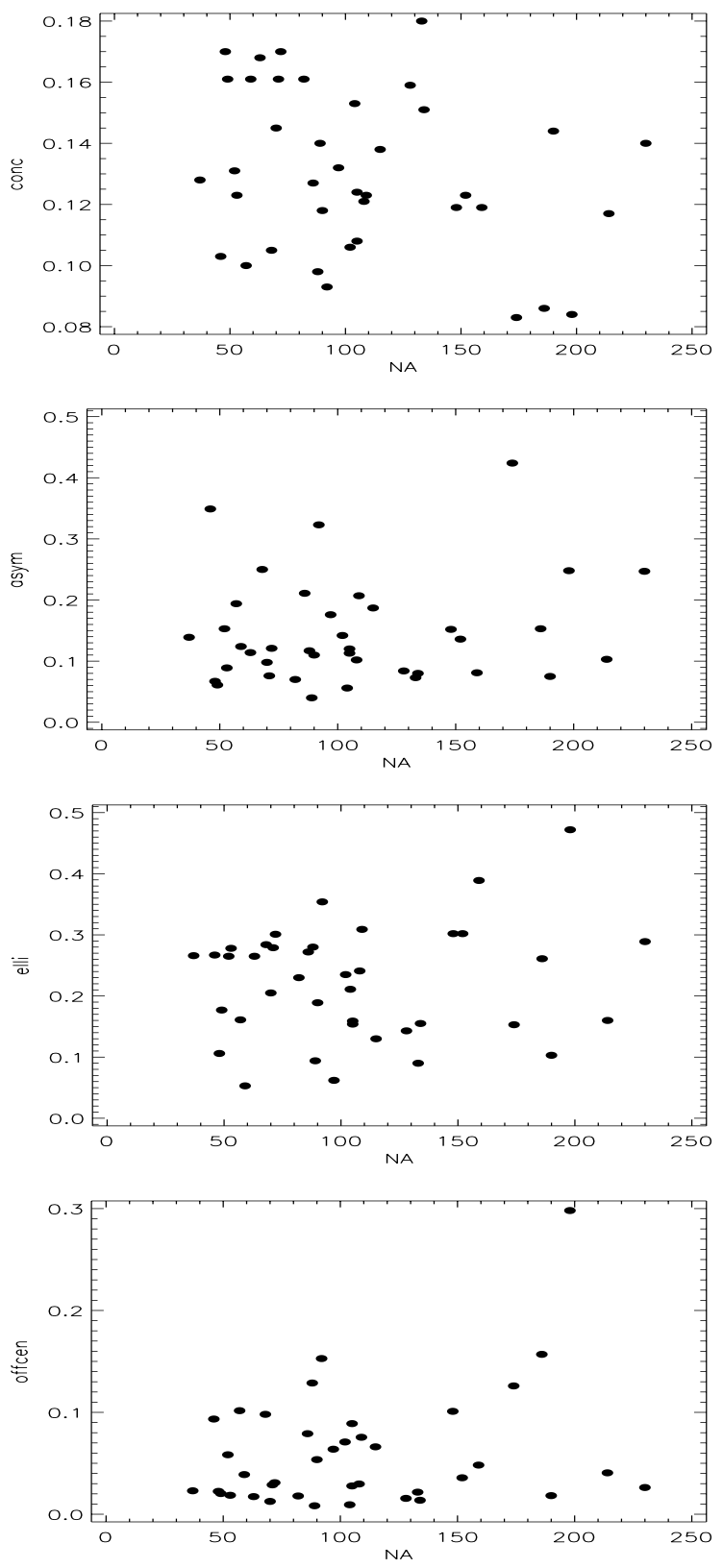

Fig. 1. Abell count (NA) compared with X-ray morphologies.

above zero are summed and then normalized by flux above the sky. We investigate the uncertainties in our X-ray measures using a Monte Carlo simulation, while cluster observations are adaptively degraded to minimize systematics caused by various exposure times, and various redshifts. (Please see Hashimoto et al. 2007 , for further details about the definition of the measures, and investigation of uncertainties and systematics.)

\section{Optical richness vs. X-ray morphology}

Figure 1 shows Abell count (NA) vs. X-ray morphologies. Abell count (NA) is Abell's (e.g. Abell et al. 1989) number count (number of galaxies within 1 Abell radius of the cluster center which are more luminous than $m_{3}+2$ mag, where $m_{3}$ is the apparent magnitude of the third most luminous cluster member; and Abell radius is the distance estimated from the magnitude of the tenth brightest cluster member. (The reader is referred to Abell's original discussion for more detail.) Abell (1958) estimated that the uncertainty in these values is $17 \%$. From our sample, 45 clusters have published NA. In Fig. 1, we see no obvious correlation between NA and X-ray morphology. The value of the rank-order correlation coefficient, Spearman $\rho$, where $\rho=1$ or -1 means a perfect linear correlation of a positive or negative slope, respectively, while $\rho=0$ indicates that two variables are uncorrelated, is $-0.26,0.08,0.04$, and 0.15 , from top to bottom panel. Corresponding significance level is $89,48,17$, and $65 \%$, respectively. The result is consistent with Hashimoto et al. (2006), where no correlation is found between X-ray morphology and X-ray luminosity or temperature of clusters, if we interpret the X-ray luminosity or temperature mainly reflects the mass of a cluster, and the richness of a cluster can be a good predictor of the mass of a cluster (Carlberg et al. 1996; Yee \& López-Cruz 1999). However, the Abell count has been known to be significantly inaccurate above redshift $z \sim 0.1$ (e.g. Southerland 1988; Struble \& Rood 1991). Therefore, to make more definitive statement, further comparison using another richness measure which is accurate above $z \sim 0.1$ is desirable.

Figure 2 shows the cluster-center galaxy correlation amplitude (Bgc) vs. X-ray morphologies. The cluster-center galaxy correlation amplitude (Bgc) is first used by Longair \& Seldner (1979) to quantify the environment of radio galaxies. Yee \& López-Cruz (1999) established the robustness (against magnitude limit, areal coverage, and photometric errors) of the Bgc as a quantitative measure of richness of galaxy clusters, with properly normalized luminosity function. They also found that there is a good correlation between Bgc and NA for Abell clusters with $z<0.1$, whereas the Abell richness classifications for Abell clusters at $z>0.1$ are much less well correlated with true cluster richness, while $\mathrm{Bgc}$ remains accurate beyond $z>0.1$. From our sample, 18 clusters, ranging from $z=0.05$ to 0.55 , have published Bgc. The Bgc values are taken from Yee \& LópezCruz (1999) and Yee \& Ellingson (2003), where their samples are selected from Abell clusters (cf. Lopez-Cruz 1997 for more detail), and Canadian Network for Observational Cosmology (CNOC1) clusters (cf. Lewis et al. 1999), respectively. In Fig. 2, we see no obvious correlation between $\mathrm{Bgc}$ and X-ray morphology. Spearman $\rho$, is $-0.31,-0.44,0.60$, and 0.04 , corresponding to $67,85,96$, and $10 \%$ significance level from top to bottom panel. The trend between Bgc vs. Elli may be significant, in such a way that richer clusters show more elongated X-ray structure. However, in general, we can see that Bgc correlates poorly to the $\mathrm{X}$-ray measures.

\section{Optical morphology vs. X-ray morphology}

Figure 3 shows a comparison between optical morphology and X-ray morphology of clusters. The optical morphology is classified by Bautz-Morgan (BM) type (Bautz \& Morgan 1970) based on visual inspection of optical images, by the degree to which the brightest cluster member stands out against the cluster background. From our sample, 25 clusters, ranging $z=0.05-0.36$, have published "certain" Bautz-Morgan type (to be on the safe side, we excluded clusters with BM type labeled "uncertain"). Figure 3 shows that X-ray morphology and optical cluster morphology by characterized by BM type is, in general, significantly correlated. The Elli vs. BM plot shows relatively stronger correlation, while other plots, show weaker correlations (Spearman $\rho$ is $-0.52,0.45,0.62$, and 0.53 , corresponding to $98.9,96.9,99.9$, and $99.1 \%$ significance level from top to bottom panel). The possible trends are, however, consistent with the fact that a cluster with big central galaxy (i.e. BM type = I) is likely to be dynam- 

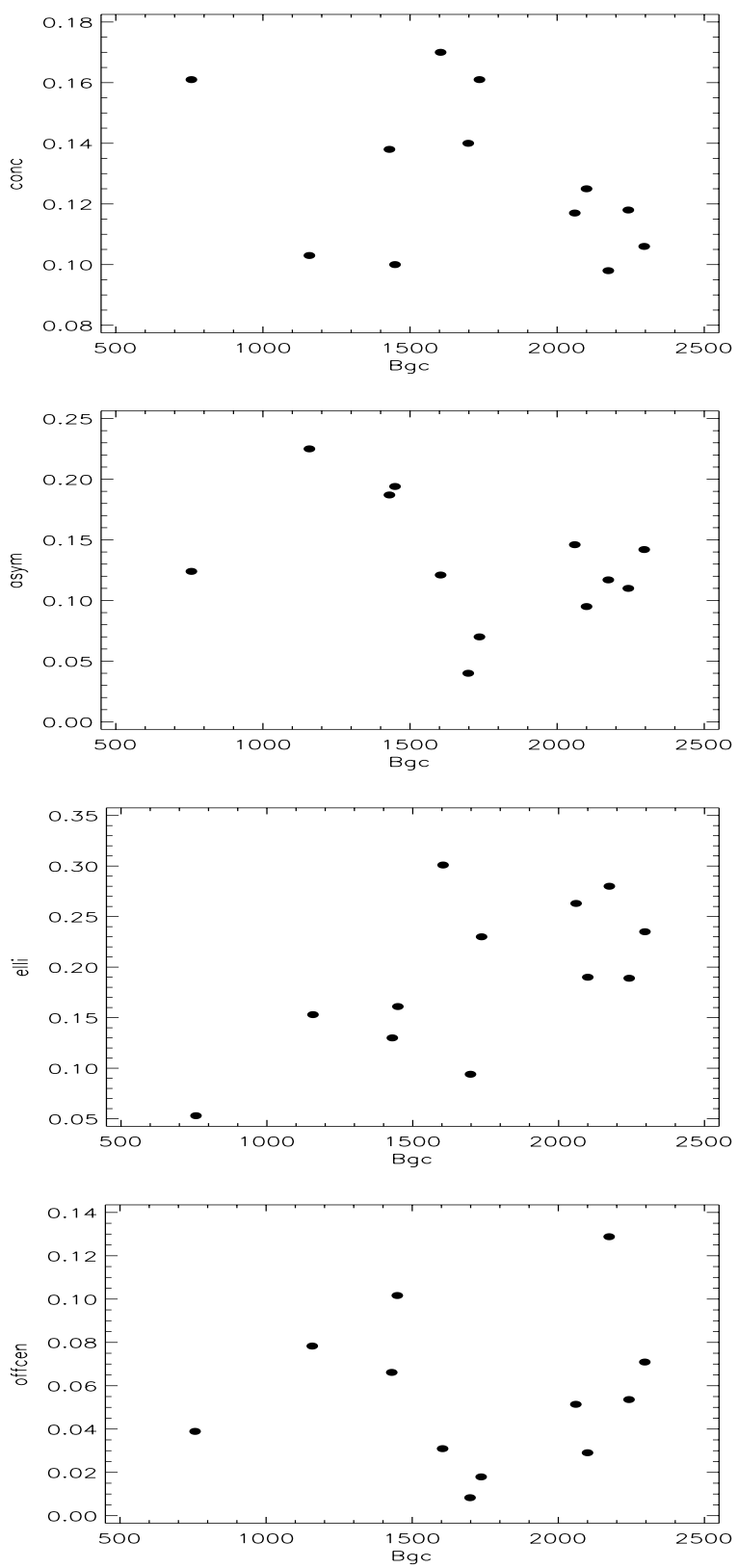

Fig. 2. The cluster-center galaxy correlation amplitude (Bgc) is compared with the X-ray morphologies.

ically settled and, therefore, showing dynamically settled X-ray morphologies, such as high concentration, small asymmetry, low ellipticity, and small degree of off-center. However, caution has to be exercised in this interpretation because, particularly for the Conc vs. BM correlation, X-ray components associated with the big central galaxy may distort the cluster X-ray morphology and produce more centrally concentrated profile.

Figure 4 shows another comparison between optical morphology and X-ray morphology of clusters. The optical morphology is now characterized by the RS system. The RS system (Rood \& Sastry 1971; Struble \& Rood 1982, 1984, 1987) is based on the projected distribution of the brightest galaxies in the cluster. The RS system is composed of six major classes: cD, B, C, L, F, and I-type clusters. These six classes have been interpreted as corresponding to a sequence of cluster evolution (Forman \& Jones 1982; Struble \& Rood 1982, 1984). The RS sample are constructed from Abell et al. (1989).
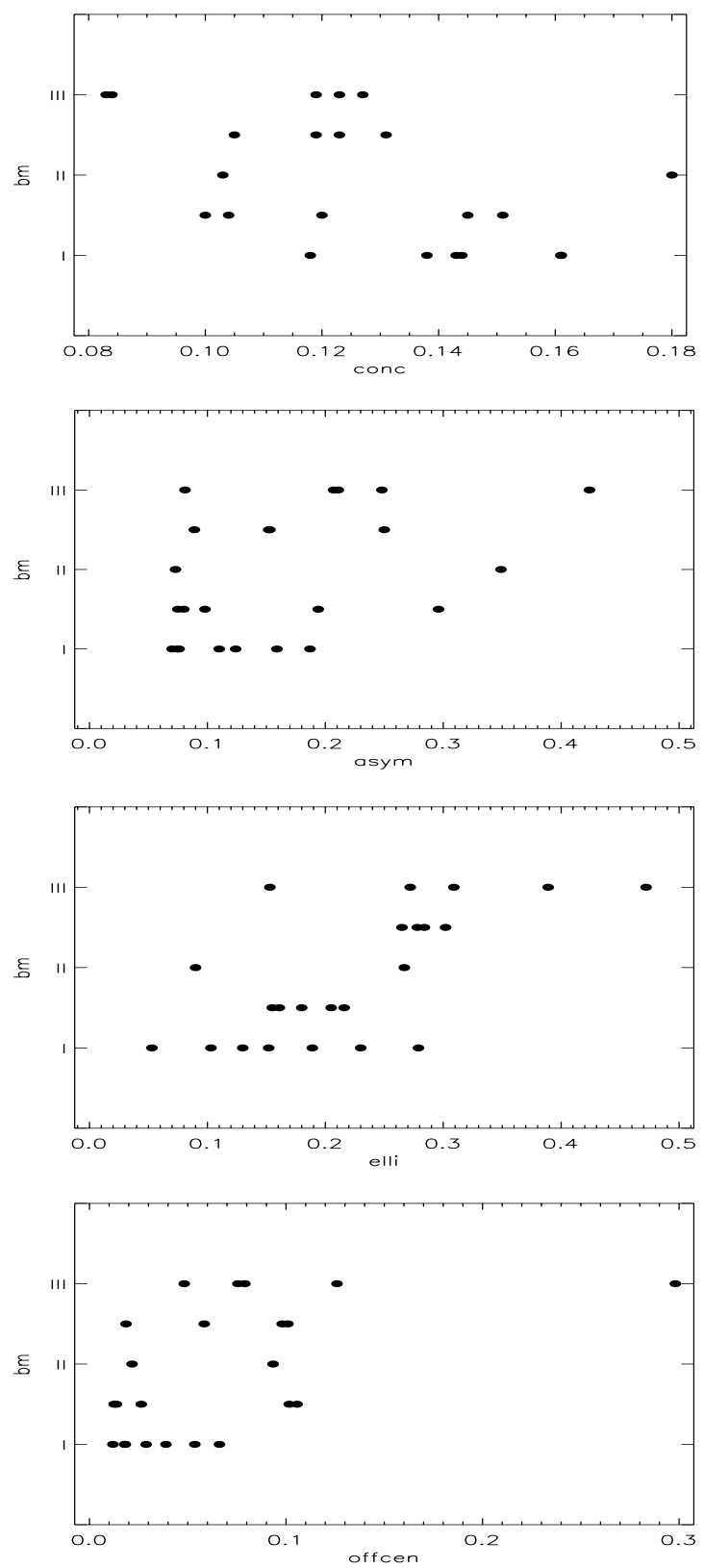

Fig. 3. A comparison between X-ray morphology and optical morphology of clusters. The optical morphology is classified by Bautz \& Morgan (1970) based on visual inspection of optical images, by the degree to which the brightest cluster member stands out against the cluster background.

From our sample, 46 clusters, ranging $z=0.05-0.36$ have published RS type. In Fig. 4, we see a wide variety of X-ray morphologies for a certain RS type, and there is no obvious correlation between the X-ray morphology and the RS type. (Spearman $\rho$ is $-0.23,0.14,0.27$, and 0.27 , corresponding to 85 , 59,90 , and $90 \%$ signficance level, from top to bottom panel). Apart from the sequence of cluster evolution, however, we see that, in Elli vs. RS type plot, clusters with optically elongated morphologies ( $\mathrm{L}$ and $\mathrm{F}$ types) tend show elongated morphologies in X-ray.

Figure 5 shows optical morphology by Butcher \& Oemler (1985) (hereafter BO) vs. X-ray morphology. BO characterized the optical morphology of clusters by the degree of central concentration of galaxy distributions, including relatively faint galaxies. The original BO sample consists of 33 clusters, 

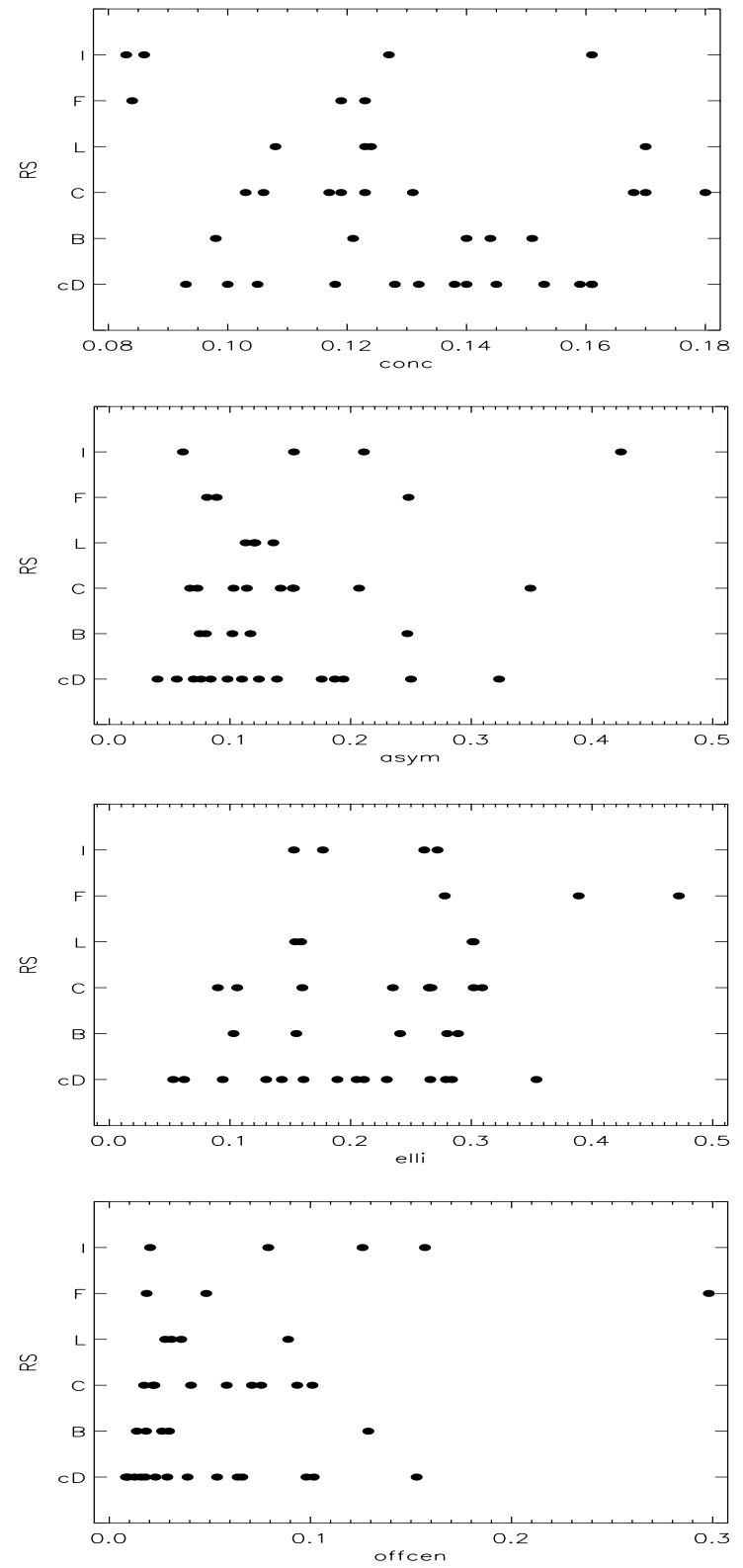

Fig. 4. A comparison between optical morphology by the RS system and X-ray morphologies. The RS system (Rood \& Sastry 1971; Struble $\&$ Rood 1982, 1984, 1987) is based on the projected distribution of the brightest galaxies in the cluster, and is composed of six major classes: $\mathrm{cD}, \mathrm{B}, \mathrm{C}, \mathrm{L}, \mathrm{F}$, and I-type clusters.

ranging in redshift from $z=0.0033$ to 0.54 . Their concentration (we refer to it as C_BO) is defined by C_BO $=\log \left(R_{60} / R_{20}\right)$ where $R_{60}$ or $R_{20}$ is the radius of the circle containing $60 \%$ or $20 \%$ of the clusters's projected galaxy distribution, respectively. Clusters whose density distributions approximate those of uniform-density spheres would have C_BO $\sim 0.3$.

From our sample, 13 clusters, ranging $z=0.06-0.54$ have published values of $\mathrm{C}_{-} \mathrm{BO}$. In Fig. 5, we see several significant correlations between $\mathrm{C} \_\mathrm{BO}$ and $\mathrm{X}$-ray morphologies, (Spearman $\rho$ is $0.41,-0.60,-0.70$, and -0.57 , corresponding to $81,96,99$, and $95 \%$ from top to bottom panel), in that a cluster with highly concentrated optical morphology is likely to be dynamically settled and, therefore, showing dynamically settled $\mathrm{X}$-ray morphologies, such as high concentration, small asymmetry, low ellipticity, and small degree of off-centerness.
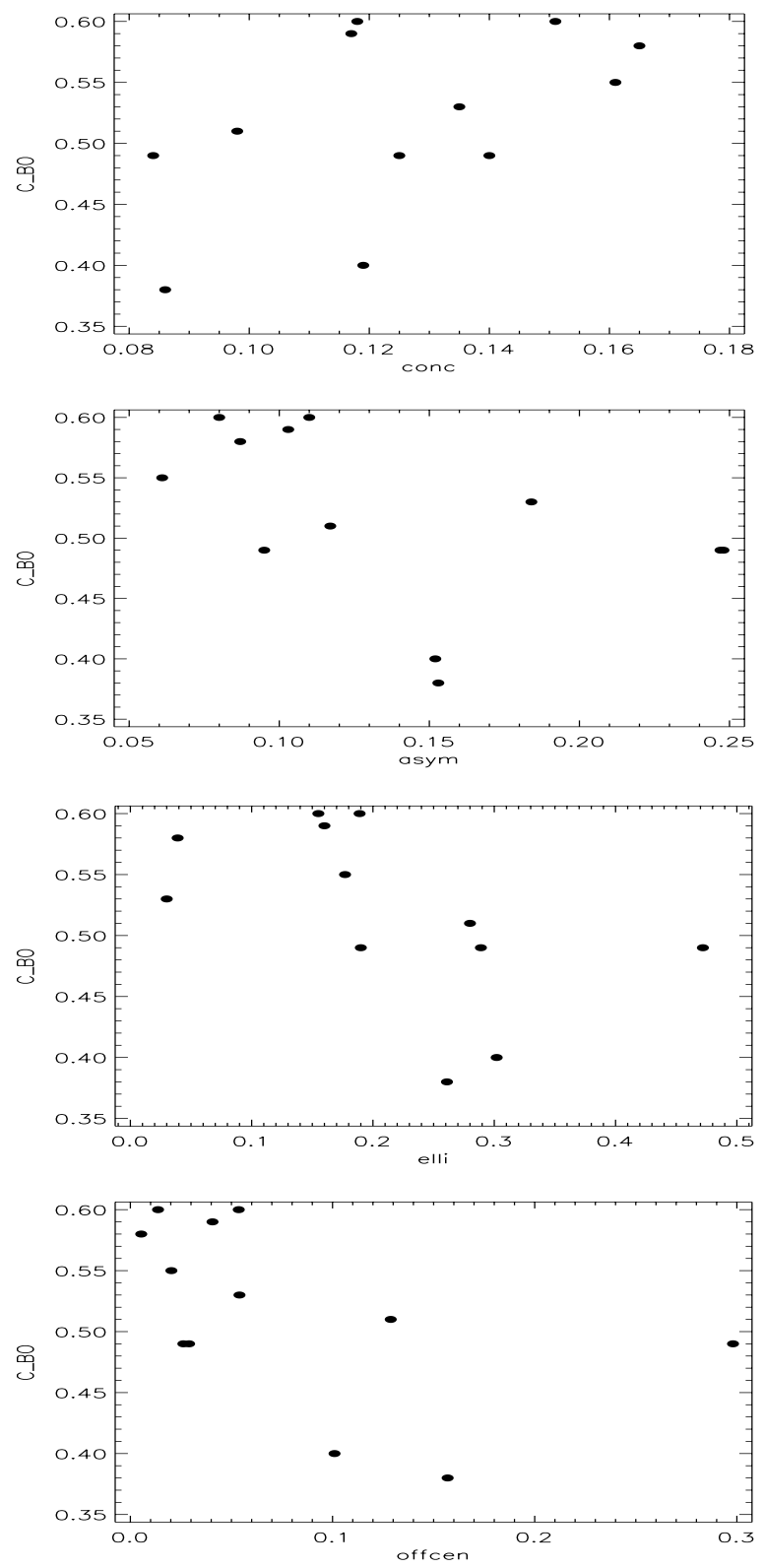

Fig. 5. The optical morphology by Butcher \& Oemler (1985) vs. X-ray morphology. Butcher \& Oemler (1985) characterized the optical morphology of clusters by the degree of central concentration of galaxy distributions. Their concentration (we refer to it as C_BO) is defined by C_BO $=\log \left(R_{60} / R_{20}\right)$ where $R_{60}$ or $\mathrm{R}_{20}$ is the radius of the circle containing $60 \%$ or $20 \%$ of the clusters's projected galaxy distribution, respectively. Clusters whose density distributions approximate those of uniform-density spheres would have C_BO $\sim 0.3$.

\section{The ratio of strong-lensing to $X$-ray mass}

Figure 6 shows the ratio of the strong-lensing to X-ray mass measurements as a function of X-ray concentration. The stronglensing mass and the X-ray mass are drawn from Allen (1998) and $\mathrm{Wu}$ (2000) (and reference herein), and are determined within the arc radii with the spherically symmetric models. The X-ray mass is calculated under the assumption of hydrostatic equilibrium and isothermal temperature profile. Note that these authors determined their temperatures by taking care of the "cooling flow" effects for high "cooling rate" clusters either by excluding the "cooling flow" region or conducting two-temperature fitting. Figure 6 demonstrates a trend (Spearman $\rho$ is -0.78 , 


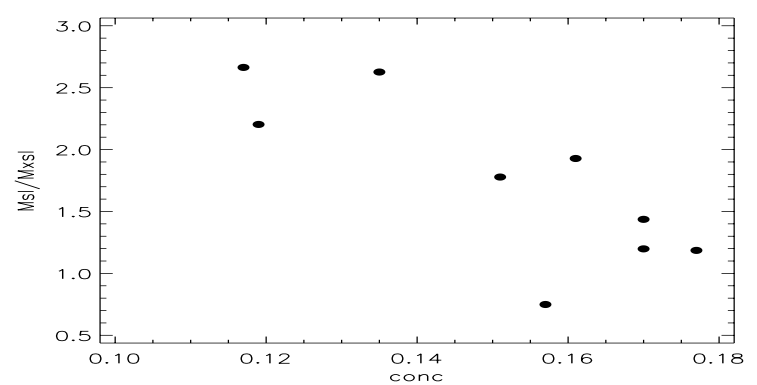

Fig. 6. The ratio of the strong-lensing to X-ray mass measurements as a function of X-ray concentration.

corresponding to $98.6 \%$ confidence level) between the mass ratio and X-ray concentration, in that clusters with high X-ray concentration show low ratio $(\sim 1)$ of the strong-lensing to $\mathrm{X}$-ray mass measurement.

This result is consistent with the interpretation that dynamically relaxed systems tend to show agreement between the strong-lensing and X-ray mass measurements, while dynamically unrelaxed systems show overestimated strong lensing mass (and/or underestimated X-ray mass). Note, however, that our other X-ray morphological measures do not show significant trends against the mass ratio. (Spearman $\rho$ is $-0.12,-0.13$, and 0.43 for Asym, Elli, and Offcen, respectively.)

\section{Conclusions and discussions}

Using a sample of 101 clusters at redshift $z \sim 0.05-1$ taken from the Chandra archive, we quantitatively investigated the relationships between the cluster X-ray morphology and various cluster characteristics determined from the optical data. The X-ray morphology is characterized by a series of objectively-measured simple statistics of X-ray surface brightness, which are designed to be robust against variations of image quality caused by various exposure times and various cluster redshifts.

The main conclusions from this work may be summarized as follows. (1) X-ray morphologies of clusters show no significant correlation with optical richness, or related quantities. (2) X-ray morphologies of clusters are significantly correlated with the degree to which the brightest cluster member stands out against the cluster background. (3) X-ray morphologies of clusters are also correlated with optical concentration defined by galaxy distribution including fainter populations. (4) The ratio of the stronglensing to X-ray mass measurements is correlated with the X-ray concentration, in that highly X-ray concentrated clusters show compatible strong-lensing and X-ray mass measurements.

The X-ray morphologies and optical morphologies defined by the degree to which the brightest cluster member stands out against the cluster background (BM system) show significant correlations overall. The correlations are consistent with the fact that a cluster with apparently dynamical-settled optical morphology shows dynamically-settled X-ray morphologies, such as high concentration, small asymmetry, low ellipticity, and small degree of off-center.

Meanwhile, optical morphologies defined by cluster surface density distribution including fainter galaxies, such as "concentration: C_BO" by Butcher \& Oemler (1985) show also a correlation with X-ray morphologies. Butcher \& Oemler (1978) noted that, at the present epoch, clusters with $\mathrm{C} \_\mathrm{BO} \sim 0.3$, which they called open or irregular clusters, have large populations of spiral galaxies, while those with $\mathrm{C} \_\mathrm{BO}>0.4$, called compact or concentrated clusters, contain few spirals in their cores. It is, therefore, possible that C_BO is actually correlated with the dynamical state of a cluster, and that is consistent with the correlation with X-ray morphologies and C_BO.

The ratio of the strong-lensing to $\mathrm{X}$-ray mass measurements is correlated with the X-ray concentration, in that highly X-ray concentrated clusters show the low mass ratio, implying the compatible strong-lensing and X-ray mass measurements. The X-ray mass measurements, or "traditional" cluster mass estimators using optical/X-ray observations of galaxies/intracluster gas, rely strongly upon the assumption of hydrostatic equilibrium, therefore the gravitational lensing serves as a powerful alternative tool for estimating masses of clusters covering a wider variety of dynamical states. It turns out that, particularly in relatively smaller scale, the strong lensing mass exceeds the X-ray mass by a factor of 2-4 (Wu 1994; Miralda-Escudé \& Babul 1995; Wu \& Fang 1997; Allen 1998). It is generally believed that such a mass discrepancy has probably arisen from either the oversimplification of strong lensing model, or the inappropriate application of hydrostatic equilibrium. However, a satisfactory explanation has not yet been given.

The fact that the ratio of the strong-lensing to X-ray mass measurements is correlated with the $\mathrm{X}$-ray concentration, in that clusters with high $X$-ray concentration show the lower ratio $(\sim 1)$, is consistent with the interpretation that dynamically relaxed systems tend to show agreement between the strong-lensing and $\mathrm{X}$-ray mass measurements, while dynamically unrelaxed systems tend to show discrepancy between the two.

The significant correlation between X-ray concentration and the mass ratio may be also consistent with previous studies (e.g. Allen 1998; Wu 2000) reporting that "cooling flow" clusters tend to produce more consistent mass estimates between strong lensing and X-ray mass measurements. Unfortunately, these studies mostly used the mass deposition rate to identify their "cooling flow" clusters. The mass deposition rate is, however, mainly based on the information from the surface brightness profile, roughly equivalent of measuring the morphology of clusters, similar to our concentration index. Thus, previously reported behaviors of the "cooling flow" clusters may be simply reflecting the "morphology effect" which is qualitatively consistent with our result, and may be showing nothing more than the fact that the clusters with high central surface brightness behave differently from those with low central surface brightness, and thus requiring different sets of assumptions for the mass estimates.

Acknowledgements. J.P.H. thanks the Alexander v. Humboldt Foundation for its We acknowledge the referee's comments, which improved the manuscript.

\section{References}

Abell, G. 1958, ApJS, 3, 211

Abell, G., Corwin, H. G., \& Olowin, R. P. 1989, ApJS, 70, 1

Allen, S. W. 1998, MNRAS, 296, 392

Bautz, L. P., \& Morgan, W. W. 1970, ApJ, 162, L149

Bird, C. 1994, AJ, 107, 1637

Böhringer, H., Voges, W., Huchra, J. P., et al. 2000, ApJS, 129, 435

Buote, D. A., \& Tsai, J. C. 1995, ApJ, 452, 522

Buote, D. A., \& Tsai, J. C. 1996, ApJ, 458, 27

Butcher, H. R., \& Oemler, A. 1985, ApJ, 285, 426

Carlberg, R. G., Yee, H. K. C., Ellingson, E., et al. 1996, ApJ, 462, 32

David, L. P., Slyz, A., Jones, C., Forman, W., Vrtilek, S. D., \& Arnaud, K. A. 1993, ApJ, 412, 479

Donahue, M., Mack, J., Scharf, C., et al. 2001, ApJ, 552, L93

Dressler, A., \& Shectman, S. A. 1988, AJ, 95, 985

Ebeling, H., Edge, A. C., Böhringer, H., et al. 1998, MNRAS, 301, 881

Ebeling, H., Edge, A. C., Allen, S. W., et al. 2000, MNRAS, 318, 333

Edge, A. C., \& Stewart, G. C. 1991, MNRAS, 252, 428

Evrard, A. E., Mohr, J. J., Fabricant, D. G., \& Geller, M. J. 1993, ApJ, 419, L9

Forman, W., \& Jones, C. 1982, ARA\&A, 20, 547 
Geller, M. J., \& Beers, T. C. 1982, PASP, 92, 421

Gioia, I. M., Maccacaro, T., Schild, R. E., et al. 1990, ApJ, 72, 567

Gomez, P. L., Pinkney, J., Burns, J. O., et al. 1997, ApJ, 474, 580

Hashimoto, Y., Oemler, A., Lin, H., \& Tucker, D. L. 1998, ApJ, 499, 589

Hashimoto, Y., Barcons, X., Böhringer, H., et al. 2004, A\&A, 419, 819

Hashimoto, Y., Böhringer, H., Henry, J. P., et al. 2007, A\&A, in press

Hasinger, G., Giacconi, R., Gunn, J. E., et al. 1998, A\&A, 340, L27

Henry, J. P., Gioia, I. M., Maccacaro, T., et al. 1992, ApJ, 386, 408

Henry, J. P., Gioia, I. M., Mullis, C. R., et al. 2001, ApJ, 553, L109

Henry, J. P., \& Henriksen, M. J. 1986, ApJ, 301, 689

Jeltema, T. E., Canizares, C. R., Bautz, M. W., \& Buote, D. A. 2005, ApJ, 624, 606

Jones, C., \& Forman, W. 1999, ApJ, 511, 65

Kolokotronis, V., Basilakos, S., Plionis, M., \& Georgabtopoulos, I. 2001, MNRAS, 320, 49

Kriessler, J. R., \& Beers, T. C. 1997, AJ, 113, 80

Lewis, A., Ellingson, E., Morris, S., et al. 1999, ApJ, 518, 533

Longair, M. S., \& Seldner, M. 1979, MNRAS, 189, 433

Lopez-Cruz, O. 1997, Ph.D Thesis, Univ. Toronto

McMillan, S. L. W., Kowalski, M. P., \& Ulmer, M. P. 1989, ApJS, 70, 723

Melott, A., Chambers, S. W., \& Miller, C. J. 2001, ApJ, 559, L75

Miralda-Escudé, J., \& Babul, A. 1995, ApJ, 449, 18

Mohr, J. J., Fabricant, D. G., \& Geller, M. J. 1993, ApJ, 413, 492
Mohr, J. J., Evrard, A. E., Fabricant, D. G., \& Geller, M. J. 1995, ApJ, 447, 8 Perlman, E. S., Horner, D. J., Jones, L. R., et al. 2002, ApJS, 140, 265 Plionis, M. 2002, ApJ, 572, L67

Popesso, P., Boehringer, H., Brinkmann, J., et al. 2004, A\&A, 423, 449 Press, W., \& Schechter, P. 1974, ApJ, 187, 425

Rhee, G. F. R. N., van Haarlem, M. P., \& Katgert, P. 1991, A\&A, 246, 301

Richstone, D., Loeb, A., \& Turner, E. L. 1992, ApJ, 393, 477

Rizza, E., Burns, J. O., Ledlow, M. J., et al. 1998, MNRAS, 301, 328

Rood, H. J., \& Sastry, G. 1971, Pub. A.S.P., 83, 313

Rosati, P., Della Ceca, R., Norman, C., \& Giacconi, R. 1998, ApJ, 492, L21

Schindler, S., Guzzo, L., Ebeling, H., et al. 1995, A\&A, 299, L9

Southerland, W. 1988, MNRAS, 234, 159

Struble, M. F., \& Rood, H. J. 1982, AJ, 87, 7

Struble, M. F., \& Rood, H. J. 1984, AJ, 89, 1487

Struble, M. F., \& Rood, H. J. 1987, ApJ, 323, 468

Struble, M. F., \& Rood, H. J. 1991, ApJ, 374, 398

Schuecker, P., Böhringer, H., Reiprich, T. H., \& Feretti, L. 2001, A\&A, 378, 408

Vikhlinin, A., McNamara, B. R., Forman, W., et al. 1998, ApJ, 502, 558

Wu, X.-P. 1994, ApJ, 436, L115

Wu, X.-P. 2000, MNRAS, 316, 299

Wu, X.-P., \& Fang, L.-Z. 1997, ApJ, 483, 62

Yee, H. K. C., \& López-Cruz, O. 1999, ApJ, 117, 1985

Yee, H. K. C., \& Ellingson, E. 2003, ApJ, 585, 215 